\title{
Extended search for supernovalike neutrinos in NOvA coincident with LIGO/Virgo detections
}

M. A. Acero, ${ }^{2}$ P. Adamson, ${ }^{12}$ L. Aliaga, ${ }^{12}$ N. Anfimov, ${ }^{26}$ A. Antoshkin,${ }^{26}$ E. Arrieta-Diaz, ${ }^{28}$ L. Asquith ${ }^{41}$ A. Aurisano, ${ }^{6}$ A. Back, ${ }^{24}$ C. Backhouse, ${ }^{46}$ M. Baird,${ }^{20,41,47}$ N. Balashov ${ }^{26}$ P. Baldi, ${ }^{25}$ B. A. Bambah, ${ }^{17}$ S. Bashar, ${ }^{45}$ K. Bays,, 19 R. Bernstein, ${ }^{12}$ V. Bhatnagar, ${ }^{34}$ B. Bhuyan, ${ }^{14}$ J. Bian, ${ }^{25,31}$ J. Blair, ${ }^{16}$ A. C. Booth,${ }^{41}$ R. Bowles, ${ }^{20}$ C. Bromberg, ${ }^{29}$ N. Buchanan, ${ }^{8}$ A. Butkevich, ${ }^{22}$ S. Calvez, ${ }^{8}$ T. J. Carroll ${ }^{44,50}$ E. Catano-Mur, ${ }^{49}$ B. C. Choudhary, ${ }^{10}$ A. Christensen, ${ }^{8}$ T. E. Coan,${ }^{39}$ M. Colo, ${ }^{49}$ L. Corwin,${ }^{51}$ L. Cremonesi,${ }^{36,46}$ G. S. Davies, ${ }^{32,20}$ P. F. Derwent, ${ }^{12}$ P. Ding, ${ }^{12}$ Z. Djurcic, ${ }^{1}$ M. Dolce, ${ }^{45}$ D. Doyle,${ }^{8}$ D. Dueñas Tonguino, ${ }^{6}$ E. C. Dukes ${ }^{47}$ H. Duyang,${ }^{38}$ S. Edayath, ${ }^{7}$ R. Ehrlich, ${ }^{47}$ M. Elkins, ${ }^{24}$ E. Ewart, ${ }^{20}$ G. J. Feldman, ${ }^{15}$ P. Filip, ${ }^{23}$ J. Franc, ${ }^{9}$ M. J. Frank ${ }^{37}$ H. R. Gallagher,${ }^{45}$ R. Gandrajula, ${ }^{29,47}$ F. Gao,${ }^{35}$ A. Giri, ${ }^{18}$ R. A. Gomes, ${ }^{13}$ M. C. Goodman, ${ }^{1}$ V. Grichine, ${ }^{27}$ M. Groh, ${ }^{8,20}$ R. Group, ${ }^{47}$ B. Guo, ${ }^{38}$ A. Habig, ${ }^{30}$ F. Hakl, ${ }^{21}$ A. Hall, ${ }^{47}$ J. Hartnell, ${ }^{41}$ R. Hatcher, ${ }^{12}$ A. Hatzikoutelis, ${ }^{43, \dagger}$ H. Hausner, ${ }^{50}$ K. Heller, ${ }^{31}$ J. Hewes, ${ }^{6}$ A. Himmel, ${ }^{12}$ A. Holin ${ }^{46}$ J. Huang, ${ }^{44}$ B. Jargowsky, ${ }^{25}$ J. Jarosz ${ }^{8}$ F. Jediny, ${ }^{9}$ C. Johnson, ${ }^{8}$ M. Judah,${ }^{8,35}$ I. Kakorin, ${ }^{26}$ D. Kalra ${ }^{34}$ D. M. Kaplan, ${ }^{19}$ A. Kalitkina, ${ }^{26}$ R. Keloth, ${ }^{7}$ O. Klimov, ${ }^{26}$ L. W. Koerner, ${ }^{16}$ L. Kolupaeva, ${ }^{26}$ S. Kotelnikov, ${ }^{27}$ R. Kralik, ${ }^{41}$ Ch. Kullenberg, ${ }^{26}$ M. Kubu, ${ }^{9}$ A. Kumar, ${ }^{34}$ C. D. Kuruppu, ${ }^{38}$ V. Kus, ${ }^{9}$ T. Lackey, ${ }^{20}$ K. Lang, ${ }^{44}$ P. Lasorak,${ }^{41}$ J. Lesmeister, ${ }^{16}$ S. Lin, ${ }^{8}$ A. Lister, ${ }^{50}$ J. Liu, ${ }^{25}$ M. Lokajicek ${ }^{23}$ S. Magill, ${ }^{1}$ M. Manrique Plata, ${ }^{20}$ W. A. Mann,${ }^{45}$ M. L. Marshak, ${ }^{31}$ M. Martinez-Casales ${ }^{24}$ V. Matveev,${ }^{22}$ B. Mayes, ${ }^{41}$ D. P. Méndez, ${ }^{41}$ M. D. Messier, ${ }^{20}$ H. Meyer, ${ }^{48}$ T. Miao, ${ }^{12}$ W. H. Miller, ${ }^{31}$ S. R. Mishra, ${ }^{38}$ A. Mislivec, ${ }^{31}$ R. Mohanta, ${ }^{17}$ A. Moren, ${ }^{30}$ A. Morozova ${ }^{26}$ W. Mu, ${ }^{12}$ L. Mualem, ${ }^{4}$ M. Muether, ${ }^{48}$ S. Mufson, ${ }^{20}$ K. Mulder ${ }^{46}$ D. Naples ${ }^{35}$ N. Nayak, ${ }^{25}$ J. K. Nelson, ${ }^{49}$ R. Nichol, ${ }^{46}$ E. Niner, ${ }^{12}$ A. Norman, ${ }^{12}$ A. Norrick, ${ }^{12}$ T. Nosek, ${ }^{5}$ H. Oh, ${ }^{6}$ A. Olshevskiy, ${ }^{26}$ T. Olson, ${ }^{45}$ J. Ott, ${ }^{25}$ J. Paley, ${ }^{12}$ R. B. Patterson, ${ }^{4}$ G. Pawloski, ${ }^{31}$ O. Petrova, ${ }^{26}$ R. Petti, ${ }^{38}$ D. D. Phan,${ }^{4,46}$ R. K. Plunkett, ${ }^{12}$ J. C. C. Porter, ${ }^{41}$ A. Rafique, ${ }^{1}$ F. Psihas,${ }^{20,44}$ V. Raj, ${ }^{4}$ M. Rajaoalisoa, ${ }^{6}$ B. Ramson, ${ }^{12}$ B. Rebel,${ }^{12,50}$ P. Rojas, ${ }^{8}$ V. Ryabov, ${ }^{27}$ O. Samoylov, ${ }^{26}$ M. C. Sanchez, ${ }^{24}$ S. Sánchez Falero, ${ }^{24}$ P. Shanahan, ${ }^{12}$ A. Sheshukov, ${ }^{26}$ P. Singh, ${ }^{10}$ V. Singh, ${ }^{3}$ E. Smith, ${ }^{20}$ J. Smolik, ${ }^{9}$ P. Snopok, ${ }^{19}$ N. Solomey, ${ }^{48}$ A. Sousa,${ }^{6}$ K. Soustruznik, ${ }^{5}$ M. Strait $\odot,{ }^{31, *}$ L. Suter, ${ }^{12}$ A. Sutton, ${ }^{47}$ S. Swain, ${ }^{33}$ C. Sweeney, ${ }^{46}$ B. Tapia Oregui, ${ }^{44}$ P. Tas, ${ }^{5}$ T. Thakore, ${ }^{6}$ R. B. Thayyullathil, ${ }^{7}$ J. Thomas, ${ }^{46,50}$ E. Tiras, ${ }^{11,24}$ J. Tripathi ${ }^{34}$ J. Trokan-Tenorio, ${ }^{49}$ A. Tsaris, ${ }^{12}$ Y. Torun, ${ }^{19}$ J. Urheim, ${ }^{20}$ P. Vahle,${ }^{49}$ Z. Vallari, ${ }^{4}$ J. Vasel, ${ }^{20}$ P. Vokac, ${ }^{9}$ T. Vrba ${ }^{9}$

M. Wallbank, ${ }^{6}$ T. K. Warburton, ${ }^{24}$ M. Wetstein, ${ }^{24}$ D. Whittington, ${ }^{42,20}$ D. A. Wickremasinghe, ${ }^{12}$ S. G. Wojcicki, ${ }^{40}$ J. Wolcott, ${ }^{45}$ W. Wu, ${ }^{25}$ Y. Xiao, ${ }^{25}$ A. Yallappa Dombara, ${ }^{42}$ K. Yonehara, ${ }^{12}$ S. Yu, ${ }^{1,19}$ Y. Yu, ${ }^{19}$ S. Zadorozhnyy, ${ }^{22}$ J. Zalesak, ${ }^{23}$ Y. Zhang, ${ }^{41}$ and R. Zwaska ${ }^{12}$

\section{(The NOvA Collaboration)}

\author{
${ }^{1}$ Argonne National Laboratory, Argonne, Illinois 60439, USA \\ ${ }^{2}$ Universidad del Atlantico, Carrera 30 No. 8-49, Puerto Colombia, Atlantico, Colombia \\ ${ }^{3}$ Department of Physics, Institute of Science, Banaras Hindu University, Varanasi, 221 005, India \\ ${ }^{4}$ California Institute of Technology, Pasadena, California 91125, USA \\ ${ }^{5}$ Charles University, Faculty of Mathematics and Physics, Institute of Particle and Nuclear Physics, \\ Prague, Czech Republic \\ ${ }^{6}$ Department of Physics, University of Cincinnati, Cincinnati, Ohio 45221, USA \\ ${ }^{7}$ Department of Physics, Cochin University of Science and Technology, Kochi 682 022, India \\ ${ }^{8}$ Department of Physics, Colorado State University, Fort Collins, Colorado 80523-1875, USA \\ ${ }^{9}$ Czech Technical University in Prague, Brehova 7, 11519 Prague 1, Czech Republic \\ ${ }^{10}$ Department of Physics and Astrophysics, University of Delhi, Delhi 110007, India \\ ${ }^{11}$ Department of Physics, Erciyes University, Kayseri 38030, Turkey \\ ${ }^{12}$ Fermi National Accelerator Laboratory, Batavia, Illinois 60510, USA \\ ${ }^{13}$ Instituto de Física, Universidade Federal de Goiás, Goiânia, Goiás, 74690-900, Brazil \\ ${ }^{14}$ Department of Physics, IIT Guwahati, Guwahati, 781 039, India \\ ${ }^{15}$ Department of Physics, Harvard University, Cambridge, Massachusetts 02138, USA \\ ${ }^{16}$ Department of Physics, University of Houston, Houston, Texas 77204, USA \\ ${ }^{17}$ School of Physics, University of Hyderabad, Hyderabad, 500 046, India \\ ${ }^{18}$ Department of Physics, IIT Hyderabad, Hyderabad, 502 205, India \\ ${ }^{19}$ Illinois Institute of Technology, Chicago, Illinois 60616, USA \\ ${ }^{20}$ Indiana University, Bloomington, Indiana 47405, USA \\ ${ }^{21}$ Institute of Computer Science, The Czech Academy of Sciences, 18207 Prague, Czech Republic \\ ${ }^{22}$ Institute for Nuclear Research of Russia, Academy of Sciences 7a, 60th October Anniversary prospect, \\ Moscow 117312, Russia
}




\author{
${ }^{23}$ Institute of Physics, The Czech Academy of Sciences, 18221 Prague, Czech Republic \\ ${ }^{24}$ Department of Physics and Astronomy, Iowa State University, Ames, Iowa 50011, USA \\ ${ }^{25}$ Department of Physics and Astronomy, University of California at Irvine, Irvine, California 92697, USA \\ ${ }^{26}$ Joint Institute for Nuclear Research, Dubna, Moscow region 141980, Russia \\ ${ }^{27}$ Nuclear Physics and Astrophysics Division, Lebedev Physical Institute, Leninsky Prospect 53, \\ 119991 Moscow, Russia \\ ${ }^{28}$ Universidad del Magdalena, Carrera 32 No 22-08 Santa Marta, Colombia \\ ${ }^{29}$ Department of Physics and Astronomy, Michigan State University, East Lansing, Michigan 48824, USA \\ ${ }^{30}$ Department of Physics and Astronomy, University of Minnesota Duluth, Duluth, Minnesota 55812, USA \\ ${ }^{31}$ School of Physics and Astronomy, University of Minnesota Twin Cities, Minneapolis, \\ Minnesota 55455, USA \\ ${ }^{32}$ University of Mississippi, University, Mississippi 38677, USA \\ ${ }^{33}$ National Institute of Science Education and Research, Khurda, 752050, Odisha, India \\ ${ }^{34}$ Department of Physics, Panjab University, Chandigarh, 160 014, India \\ ${ }^{35}$ Department of Physics, University of Pittsburgh, Pittsburgh, Pennsylvania 15260, USA \\ ${ }^{36}$ School of Physics and Astronomy, Queen Mary University of London, London E1 4NS, United Kingdom \\ ${ }^{37}$ Department of Physics, University of South Alabama, Mobile, Alabama 36688, USA \\ ${ }^{38}$ Department of Physics and Astronomy, University of South Carolina, Columbia, \\ South Carolina 29208, USA \\ ${ }^{39}$ Department of Physics, Southern Methodist University, Dallas, Texas 75275, USA \\ ${ }^{40}$ Department of Physics, Stanford University, Stanford, California 94305, USA \\ ${ }^{41}$ Department of Physics and Astronomy, University of Sussex, Falmer, \\ Brighton BN1 9QH, United Kingdom \\ ${ }^{42}$ Department of Physics, Syracuse University, Syracuse, New York 13210, USA \\ ${ }^{43}$ Department of Physics and Astronomy, University of Tennessee, Knoxville, Tennessee 37996, USA \\ ${ }^{44}$ Department of Physics, University of Texas at Austin, Austin, Texas 78712, USA \\ ${ }^{45}$ Department of Physics and Astronomy, Tufts University, Medford, Massachusetts 02155, USA \\ ${ }^{46}$ Physics and Astronomy Department, University College London, Gower Street, \\ London WC1E 6BT, United Kingdom \\ ${ }^{47}$ Department of Physics, University of Virginia, Charlottesville, Virginia 22904, USA \\ ${ }^{48}$ Department of Mathematics, Statistics, and Physics, Wichita State University, \\ Wichita, Kansas 67206, USA \\ ${ }^{49}$ Department of Physics, William \& Mary, Williamsburg, Virginia 23187, USA \\ ${ }^{50}$ Department of Physics, University of Wisconsin-Madison, Madison, Wisconsin 53706, USA \\ ${ }^{51}$ South Dakota School of Mines and Technology, Rapid City, South Dakota 57701, USA
}

(Received 10 June 2021; accepted 16 August 2021; published 15 September 2021)

A search is performed for supernovalike neutrino interactions coincident with 76 gravitational wave events detected by the LIGO/Virgo Collaboration. For 40 of these events, full readout of the time around the gravitational wave is available from the NOvA Far Detector. For these events, we set limits on the fluence of the sum of all neutrino flavors of $F<7(4) \times 10^{10} \mathrm{~cm}^{-2}$ at $90 \%$ C.L. assuming energy and time distributions corresponding to the Garching supernova models with masses 9.6(27) $\mathrm{M}_{\odot}$. Under the hypothesis that any given gravitational wave event was caused by a supernova, this corresponds to a distance of $r>29(50) \mathrm{kpc}$ at $90 \%$ C.L. Weaker limits are set for other gravitational wave events with partial Far Detector data and/or Near Detector data.

DOI: 10.1103/PhysRevD.104.063024

\title{
I. INTRODUCTION
}

* Corresponding author.

straitm@umn.edu

Present address: San José State University.

Published by the American Physical Society under the terms of the Creative Commons Attribution 4.0 International license. Further distribution of this work must maintain attribution to the author(s) and the published article's title, journal citation, and DOI. Funded by SCOAP ${ }^{3}$.
Multimessenger astronomy is a rapidly expanding field, with exciting opportunities to simultaneously observe violent astrophysical events using gravitational waves (GWs), electromagnetic radiation, cosmic rays, and neutrinos. To date, a single gravitational wave event has been associated with electromagnetic activity [1-3], and none have been associated with the other channels. Not all gravitational waves and gravitational wave candidates to 
date have been identified by the LIGO/Virgo Collaboration (LVC) with a particular production mechanism [4]. Although all clearly identified events are associated with compact object mergers, there remains the possibility that one or more were caused by a supernova, which are expected to produce gravitational waves, but with great uncertainty in predictions of the signal strength [5]. These potential supernovae may have evaded optical detection either because they were obscured by dust in the central Galaxy, or because they were "failed" supernovae in which the star collapsed, but did not explode [6].

In a previous paper [7] we described a broad search for signals, across the $\mathrm{MeV}$ to $\mathrm{TeV}$ range, associated with 26 gravitational wave events. We now focus on the possibility of detecting supernovalike neutrinos and present an improved search using the now-available larger catalog of gravitational wave events. The paper is organized as follows. In Sec. II, we introduce the NOvA detectors. Section III details the data set used in this analysis. Section IV explains how we simulate supernova neutrino interactions. Section V describes the improved selection of supernovalike neutrinos. Finally, Sec. VI gives the results.

\section{DETECTORS}

The NOvA experiment consists of two similar detectors, the Near Detector (ND) and the Far Detector (FD). The ND is located at the Fermi National Accelerator Laboratory (Fermilab), $100 \mathrm{~m}$ underground, while the FD is located near Ash River, Minnesota, on the surface with a modest overburden consisting of $1.25 \mathrm{~m}$ of concrete covered with $16 \mathrm{~cm}$ of barite gravel.

The NOvA detectors are segmented liquid scintillator tracking calorimeters. Alternating planes of cells are oriented horizontally and vertically, forming two views that can be used to reconstruct three-dimensional positions. The cells have a cross section of $4 \mathrm{~cm}$ by $6 \mathrm{~cm}$ and are $15.5 \mathrm{~m}(3.8 \mathrm{~m})$ long in the FD (ND). The FD has 896 planes of cells and a total mass of $14 \mathrm{kt}$, whereas the ND has 214 planes and a total mass of $300 \mathrm{t}$. The last 20 planes at the north end of the ND are a muon catcher. They are interleaved with ten $10-\mathrm{cm}$-thick planes of steel for the purpose of measuring the energy of muons produced in beam interactions. The FD has no similar structure. The detectors are described in more detail elsewhere [8].

Light produced in the scintillator is collected by wavelength-shifting fibers and converted into electrical signals using avalanche photodiodes. These signals are continuously digitized at $2 \mathrm{MHz}$ at the FD and $8 \mathrm{MHz}$ at the ND. Samples rising above a threshold, called hits, are retained for further processing. Hits from all channels are collected into $50 \mu$ s blocks and can be saved for offline analysis if a software trigger requests them within about 20 minutes for the Far Detector and 30 minutes at the Near Detector. Triggers can either be based on the content of the data or on external signals. Two of the latter type of triggers are used in this analysis. First, when LVC publishes an observation of a gravitational wave candidate over the Gamma-ray Coordinates Network, we respond by reading out $45 \mathrm{~s}$ of continuous data from both the ND and FD, beginning $5.16 \mathrm{~s}$ prior to the gravitational wave time stamp. Second, we run a minimum bias pulser trigger on the FD which reads out $550 \mu$ s segments of data at a rate of $10 \mathrm{~Hz}$. When only pulser data is available, we use a window of $1000 \mathrm{~s}$ centered on the gravitational wave time stamp to match the convention established by other neutrino observatories $[9,10]$.

The NOvA detectors are exposed to Fermilab's NuMI beam [11], a wideband neutrino beam with a peak at $2 \mathrm{GeV}$ consisting mainly of either $\nu_{\mu}$ or $\bar{\nu}_{\mu}$, depending on the operating mode. Typically, the beam is operated October through June with pulses of $10 \mu \mathrm{s}$ separated by $1.3 \mathrm{~s}$. For the purposes of the analysis reported here, the beam has no impact on the FD data since the number of beam neutrino interactions is negligible. However, it is a source of background at the ND; a procedure to remove beam backgrounds is detailed in Sec. V.

\section{DATA SET}

Tables I and II show a summary of NOvA data collected for each of the gravitational wave events and candidates (henceforth called "events") announced by LVC to date in their two catalogs [4,12] and via the Gamma-ray Coordinates Network [13-42]. With the exception of four gravitational wave events, at least one of the NOvA detectors was operating and taking useful data for each event. LVC issued public triggers beginning with their "O3" run period in 2019; prior to that point, NOvA has only the FD pulser data. Thirteen events in $\mathrm{O} 3$ were only announced in the second LVC catalog and not via public trigger; we only have FD pulser data for these as well.

Of the remaining $52 \mathrm{GW}$ events that did have public triggers, we recorded all or part of the desired $45 \mathrm{~s}$ of continuous data at the FD for 32 events, and at the ND for 40. In five cases, the ND recorded full readouts when the FD did not because it has a deeper data buffer. At each detector, data is read out approximately in time order; alerts that arrived when the data was near the end of the buffer resulted in partial readouts, as shown in the table. In the remaining three cases, the FD was down and the ND was up.

\section{SIMULATION}

Supernova neutrino interactions are simulated for use in training the selector and for assessing signal significance. The simulation is based on the Garching $9.6 \mathrm{M}_{\odot}$ and $27 \mathrm{M}_{\odot}$ supernova flux models [43], with neutrino interactions produced with GENIE v3.0.6 [44], and the resulting particles tracked through the detector geometry using GEANT4 v10.4.2 [45]. The simulation only includes neutrinos above $10 \mathrm{MeV}$, with inverse beta decay on hydrogen (IBD) 
TABLE I. Summary of NOvA data taking during GW events [4,12-42] and 90\% C.L. limits. The fluence limits on the two supernova models are in units of $10^{10} \mathrm{~cm}^{-2}$. The distance limits are in kiloparsecs. When continuous data was read out in response to an LVC trigger, the number of seconds read is given for each detector. Otherwise ("untriggered"), pulser data is used in the case of the FD, and the ND is not used. In some cases one or both detectors were not running ("no data") and in two cases the FD was running, but not taking good data ("bad"). Events above the line have been considered by NOvA before; above and below the line events are arranged chronologically.

\begin{tabular}{|c|c|c|c|c|c|c|}
\hline \multirow[b]{2}{*}{ Name } & \multirow[b]{2}{*}{ ND } & \multirow[b]{2}{*}{ FD } & \multicolumn{2}{|c|}{ Fluence } & \multicolumn{2}{|c|}{ Distance } \\
\hline & & & $\mathrm{SN}_{27 \odot}$ & $\mathrm{SN}_{9.6 \odot}$ & $\mathrm{SN}_{27 \odot}$ & $\mathrm{SN}_{9.6 \odot}$ \\
\hline GW150914 & Untriggered & $\mathrm{Bad}$ & $\ldots$ & $\ldots$ & $\ldots$ & $\cdots$ \\
\hline GW151012 & Untriggered & No data & $\ldots$ & $\ldots$ & $\ldots$ & $\ldots$ \\
\hline GW151226 & Untriggered & Untriggered & 110 & 190 & 9 & 6 \\
\hline GW170104 & Untriggered & Untriggered & 300 & 500 & 6 & 3.4 \\
\hline GW170608 & Untriggered & Untriggered & 400 & 700 & 5 & 2.9 \\
\hline GW170729 & Untriggered & Untriggered & 240 & 400 & 6 & 4 \\
\hline GW170809 & Untriggered & Untriggered & 110 & 190 & 9 & 6 \\
\hline GW170814 & Untriggered & Untriggered & 120 & 200 & 9 & 5 \\
\hline GW170817 & Untriggered & Untriggered & 110 & 190 & 9 & 6 \\
\hline GW170818 & Untriggered & Untriggered & 180 & 330 & 7 & 4 \\
\hline GW170823 & Untriggered & Untriggered & 260 & 500 & 6 & 3.5 \\
\hline GW190408_181802 & No data & No data & $\ldots$ & $\ldots$ & $\ldots$ & $\ldots$ \\
\hline GW190412 & Untriggered & Untriggered & 170 & 280 & 7 & 4 \\
\hline GW190421_213856 & Untriggered & Untriggered & 210 & 400 & 7 & 4 \\
\hline GW190425 & Untriggered & Untriggered & 120 & 190 & 9 & 5 \\
\hline GW190426_152155 & $44.7 \mathrm{~s}$ & Untriggered & 13 & 19 & 27 & 17 \\
\hline GW190503_185404 & Untriggered & Untriggered & 150 & 270 & 8 & 5 \\
\hline $\mathrm{S} 190510 \mathrm{~g}$ & Untriggered & Untriggered & 170 & 280 & 7 & 4 \\
\hline GW190512_180714 & Untriggered & Untriggered & 190 & 330 & 7 & 4 \\
\hline GW190513_205428 & $24.7 \mathrm{~s}$ & Untriggered & 14 & 20 & 26 & 17 \\
\hline GW190517_055101 & Untriggered & Untriggered & 120 & 200 & 9 & 5 \\
\hline GW190519_153544 & Untriggered & Untriggered & 140 & 250 & 8 & 5 \\
\hline GW190521 & $45.0 \mathrm{~s}$ & $45.0 \mathrm{~s}$ & 6 & 10 & 40 & 24 \\
\hline GW190521_074359 & Untriggered & Untriggered & 170 & 280 & 7 & 4 \\
\hline GW190602_175927 & $45.0 \mathrm{~s}$ & $45.0 \mathrm{~s}$ & 6 & 12 & 40 & 22 \\
\hline GW190630_185205 & $45.0 \mathrm{~s}$ & $45.0 \mathrm{~s}$ & 5 & 9 & 40 & 25 \\
\hline GW190701_203306 & $45.0 \mathrm{~s}$ & $45.0 \mathrm{~s}$ & 6 & 11 & 40 & 23 \\
\hline GW190706_222641 & $45.0 \mathrm{~s}$ & $17.5 \mathrm{~s}$ & 2.5 & 5 & 60 & 35 \\
\hline GW190707_093326 & Untriggered & Untriggered & 220 & 400 & 6 & 4 \\
\hline GW190413_052954 & Untriggered & Untriggered & 170 & 280 & 7 & 4 \\
\hline GW190413_134308 & Untriggered & Untriggered & 160 & 270 & 8 & 5 \\
\hline GW190424_180646 & Untriggered & Untriggered & 140 & 240 & 8 & 5 \\
\hline GW190514_065416 & Untriggered & Untriggered & 280 & 500 & 6 & 3.5 \\
\hline GW190527_092055 & Untriggered & Untriggered & 140 & 240 & 8 & 5 \\
\hline GW190620_030421 & Untriggered & Untriggered & 270 & 400 & 6 & 4 \\
\hline GW190708_232457 & Untriggered & Untriggered & 150 & 270 & 8 & 5 \\
\hline S190718y & $18.3 \mathrm{~s}$ & Untriggered & 17 & 23 & 23 & 16 \\
\hline GW190719_215514 & Untriggered & $\mathrm{Bad}$ & $\ldots$ & $\ldots$ & $\ldots$ & $\ldots$ \\
\hline GW190720_000836 & $45.0 \mathrm{~s}$ & $45.0 \mathrm{~s}$ & 4 & 6 & 50 & 31 \\
\hline GW190727_060333 & $45.0 \mathrm{~s}$ & $45.0 \mathrm{~s}$ & 5 & 9 & 40 & 25 \\
\hline
\end{tabular}

and electron elastic scattering (ES) interactions included. Since NOvA is hydrocarbon-based, IBD strongly dominates over ES. IBD is the most important interaction for NOvA because it has a large cross section and produces a high-energy positron. The mean positron energy produced in the 9.6(27) $\mathrm{M}_{\odot}$ simulation is $19.0(21.2) \mathrm{MeV}$.

In IBD interactions, both positrons and neutrons are simulated. Although NOvA is primarily sensitive to positrons and electrons, the $8 \mathrm{MeV}$ of gammas from neutron capture on ${ }^{35} \mathrm{Cl}$ is also visible. The NOvA detectors are $16 \%$ chlorine by mass. After selection cuts, the FD has no significant sensitivity to electrons and positrons below $10 \mathrm{MeV}$; however, the ND is still marginally sensitive at this energy, so the simulation somewhat undercounts the neutrino interactions that would be selected in a real supernova. 
TABLE II. Continuation of Table I.

\begin{tabular}{|c|c|c|c|c|c|c|}
\hline \multirow[b]{2}{*}{ Name } & \multirow[b]{2}{*}{ ND } & \multirow[b]{2}{*}{ FD } & \multicolumn{2}{|c|}{ Fluence } & \multicolumn{2}{|c|}{ Distance } \\
\hline & & & $\mathrm{SN}_{27 \odot}$ & $\mathrm{SN}_{9.6 \odot}$ & $\mathrm{SN}_{27 \odot}$ & $\mathrm{SN}_{9.6 \odot}$ \\
\hline GW190728_064510 & $45.0 \mathrm{~s}$ & $29.6 \mathrm{~s}$ & 3.2 & 5 & 50 & 33 \\
\hline GW190731_140936 & Untriggered & Untriggered & 210 & 400 & 7 & 4 \\
\hline GW190803_022701 & Untriggered & Untriggered & 140 & 230 & 8 & 5 \\
\hline GW190814 & $45.0 \mathrm{~s}$ & Untriggered & 14 & 22 & 25 & 16 \\
\hline GW190828_063405 & $45.0 \mathrm{~s}$ & $18.1 \mathrm{~s}$ & 6 & 10 & 40 & 23 \\
\hline GW190828_065509 & $45.0 \mathrm{~s}$ & Untriggered & 16 & 21 & 24 & 16 \\
\hline S190901ap & $45.0 \mathrm{~s}$ & $45.0 \mathrm{~s}$ & 3.1 & 6 & 60 & 30 \\
\hline GW190909_114149 & Untriggered & Untriggered & 110 & 190 & 9 & 5 \\
\hline S190910d & $45.0 \mathrm{~s}$ & $45.0 \mathrm{~s}$ & 4 & 7 & 50 & 29 \\
\hline S190910h & $45.0 \mathrm{~s}$ & $45.0 \mathrm{~s}$ & 2.7 & 5 & 60 & 32 \\
\hline GW190910_112807 & Untriggered & Untriggered & 120 & 190 & 9 & 6 \\
\hline GW190915_235702 & $45.0 \mathrm{~s}$ & $45.0 \mathrm{~s}$ & 3.0 & 6 & 60 & 31 \\
\hline S190923y & $45.0 \mathrm{~s}$ & $45.0 \mathrm{~s}$ & 3.2 & 6 & 50 & 32 \\
\hline GW190924_021846 & $45.0 \mathrm{~s}$ & $45.0 \mathrm{~s}$ & 4 & 7 & 50 & 28 \\
\hline GW190929_012149 & Untriggered & Untriggered & 200 & 340 & 7 & 4 \\
\hline GW190930_133541 & $45.0 \mathrm{~s}$ & $45.0 \mathrm{~s}$ & 7 & 13 & 40 & 21 \\
\hline S190930t & $45.0 \mathrm{~s}$ & $45.0 \mathrm{~s}$ & 5 & 10 & 40 & 23 \\
\hline S191105e & Untriggered & Untriggered & 180 & 310 & 07 & 4 \\
\hline S191109d & $45.0 \mathrm{~s}$ & $45.0 \mathrm{~s}$ & 5 & 8 & 40 & 26 \\
\hline S191129u & Untriggered & Untriggered & 230 & 400 & 06 & 4 \\
\hline S191204r & Untriggered & Untriggered & 300 & 500 & 06 & 3.4 \\
\hline S191205ah & $45.0 \mathrm{~s}$ & $45.0 \mathrm{~s}$ & 2.7 & 6 & 60 & 32 \\
\hline S191213g & $45.0 \mathrm{~s}$ & $45.0 \mathrm{~s}$ & 3.4 & 7 & 50 & 29 \\
\hline S191215w & $45.0 \mathrm{~s}$ & $45.0 \mathrm{~s}$ & 4 & 7 & 50 & 29 \\
\hline S191216ap & $45.0 \mathrm{~s}$ & $29.5 \mathrm{~s}$ & 02.7 & 05 & 60 & 35 \\
\hline S191222n & $45.0 \mathrm{~s}$ & $45.0 \mathrm{~s}$ & 4 & 7 & 50 & 29 \\
\hline S200105ae & Untriggered & Untriggered & 230 & 400 & 06 & 4 \\
\hline S200112r & $45.0 \mathrm{~s}$ & No data & 16 & 23 & 24 & 16 \\
\hline S200114f & $45.0 \mathrm{~s}$ & $45.0 \mathrm{~s}$ & 9 & 15 & 32 & 19 \\
\hline S200115j & $45.0 \mathrm{~s}$ & $45.0 \mathrm{~s}$ & 2.1 & 4 & 70 & 40 \\
\hline S200128d & $45.0 \mathrm{~s}$ & $45.0 \mathrm{~s}$ & 5 & 8 & 50 & 26 \\
\hline S200129m & $45.0 \mathrm{~s}$ & $45.0 \mathrm{~s}$ & 3.2 & 6 & 50 & 32 \\
\hline S200208q & $45.0 \mathrm{~s}$ & $45.0 \mathrm{~s}$ & 5 & 7 & 40 & 28 \\
\hline S200213t & $45.0 \mathrm{~s}$ & $45.0 \mathrm{~s}$ & 5 & 10 & 40 & 23 \\
\hline S200219ac & Untriggered & Untriggered & 190 & 300 & 07 & 4 \\
\hline S200224ca & $45.0 \mathrm{~s}$ & No data & 22 & 29 & 21 & 14 \\
\hline S200225q & $45.0 \mathrm{~s}$ & $45.0 \mathrm{~s}$ & 3.4 & 6 & 50 & 30 \\
\hline S200302c & $45.0 \mathrm{~s}$ & $45.0 \mathrm{~s}$ & 4 & 8 & 50 & 26 \\
\hline S200311bg & $45.0 \mathrm{~s}$ & No data & 16 & 21 & 24 & 16 \\
\hline S200316bj & $45.0 \mathrm{~s}$ & $45.0 \mathrm{~s}$ & 2.9 & 5 & 60 & 33 \\
\hline
\end{tabular}

Besides undercounting low-energy neutrino interactions, the simulation also does not include various interaction channels on carbon such as $\nu_{e}+{ }^{12} \mathrm{C} \rightarrow e^{-}+{ }^{12} \mathrm{~N}$, nor similar channels involving other isotopes in the NOvA materials, although in many cases, these interactions would be easily visible. The limits set below are therefore conservative, although IBD would dominate over these other channels even if they were included. We use a model without neutrino oscillations or other flavor-changing effects because there is not enough information available to know whether these effects would increase or decrease the number of neutrinos observed by NOvA [43].

\section{ANALYSIS}

Relative to our previous report [7], the clustering algorithm for grouping hits into supernova neutrino event candidates has been greatly improved. Previously, a cluster was defined as a pair of hits with one hit in each view. Now a cluster may have two to seven hits associated in time and space. Clusters of greater than seven hits are rejected as being too large to have been produced by a supernova neutrino interaction. In the ND, we allow clusters with all hits in a single view. However, at the FD, three-dimensional position information is essential for reducing background, so clusters must include hits in both views. Similarly, ND 
clusters may be noncontiguous, with gaps either between hits within a detector plane or between detector planes, but FD clusters must be contiguous to reduce background. Previously, we excluded the muon catcher region of the ND; clusters in this region are now accepted.

Critical to the reduction of background, particularly at the FD, is the inclusion of several new variables in the classifier that relate the distance in time and space between candidate hit clusters and recent cosmic rays. Michel electrons from stopping muons are a common background in the FD, occurring at a rate of $40 \mathrm{kHz}$. Most Michel electrons are identified by close association with track ends, but a small fraction of apparent Michel electrons appear far from the track end, either because of reconstruction failures, inefficiencies in producing hits, complex particle interactions, or some combination of these. Candidate clusters are judged based on their proximity to the track end, to any point along the track, as well as to any hit in a large cluster of activity with no reconstructed tracks.

Supernova neutrino-like hit clusters are separated into signal and background samples using the scikit-learn [46] package's RandomForestClassifier class. The classifier is trained with simulated $9.6 \mathrm{M}_{\odot}$ supernova interactions and real minimum-bias data from the NOvA detectors. The classifier was optimized separately for the ND and the FD. Further, it is optimized separately for the two cases of FD data: continuous readout and pulser. The pulser data must be treated differently because the lookback time for cosmic rays that may have produced a background cluster is reduced. Additionally, since the live time is smaller, efficiency is prioritized over background reduction. In all three cases, the figure of merit [47]

$$
\frac{\text { signal }}{a / 2+\sqrt{\text { background }}},
$$

is optimized, with $a=1.292$ to optimize $90 \%$ C.L. limits. The resulting efficiencies for IBD positrons are shown in Fig. 1. Efficiencies for ES electrons, as a function of electron energy, are very similar. At the ND, neutron captures from IBD are selected with $2 \%$ efficiency, while at the FD the neutron capture efficiency is negligible for purposes of the signal: only $0.02 \%$. No attempt is made in the analysis to associate positron and neutron delayed coincidences in either detector.

Compared with our previous analysis method, the rate of selected background candidates in the FD, for continuous readout, is reduced by a factor of 80 , from 460 to $6 \mathrm{~Hz}$, while the signal efficiency for IBD positrons is reduced from $7.8 \%$ to $4.3 \%$. The reduced signal efficiency is a consequence of the optimization described above. In the previous analysis, the same selection was used for FD continuous-readout and FD pulser data. In this analysis, the pulser background rate is reduced to $55 \mathrm{~Hz}$, while the signal efficiency is increased to $9.0 \%$, or $0.3 \mathrm{~Hz}$ and $0.05 \%$ taking into account the $0.55 \%$ live time. In the ND, the rate of
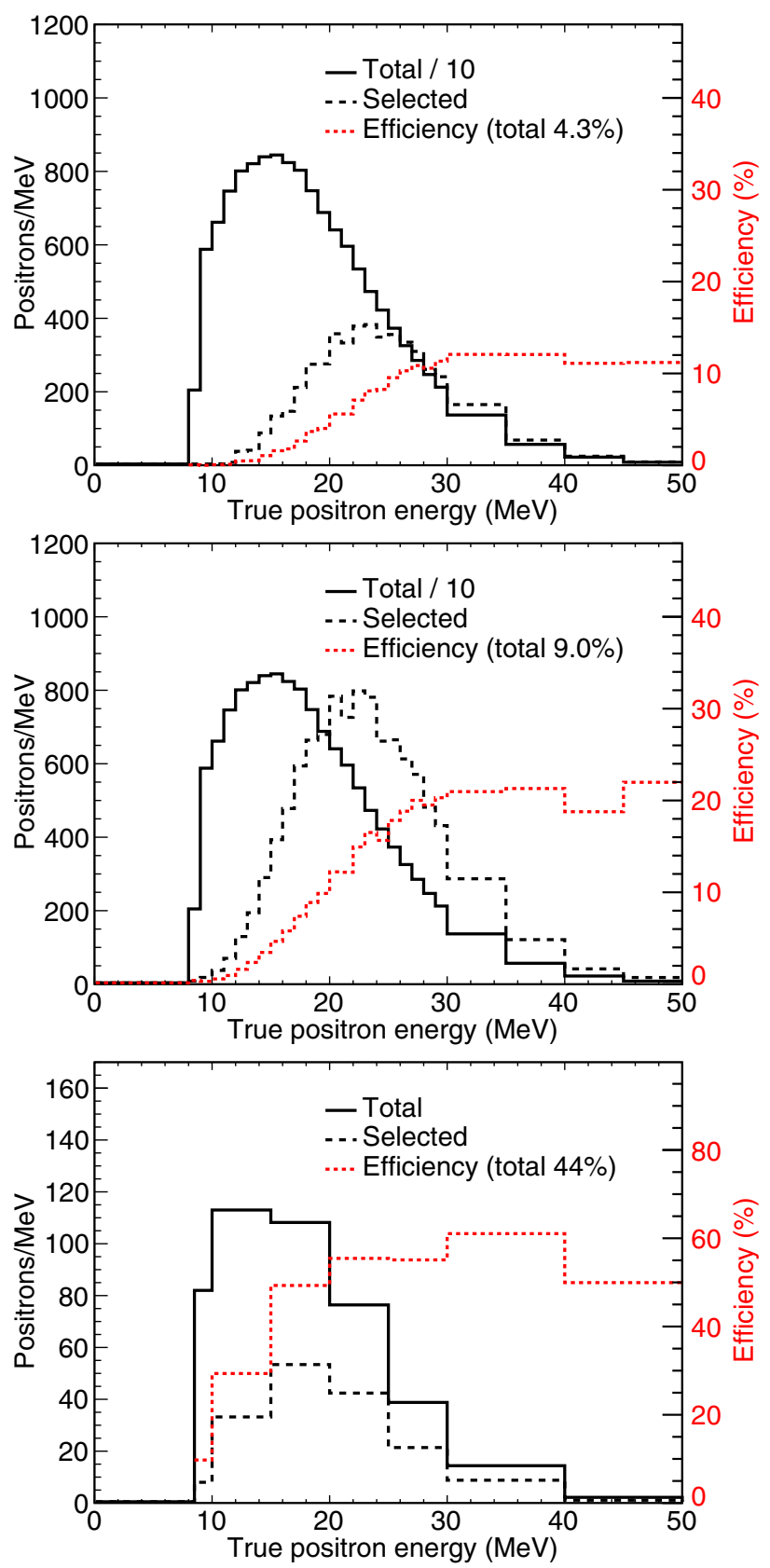

FIG. 1. Top (middle, bottom): FD continuous readout (FD pulser, ND) total and selected positron spectra (left axis) and positron selection efficiency (right axis) as a function of energy. Each plot shows a simulated $9.6 \mathrm{M}_{\odot}$ supernova at $1 \mathrm{kpc}$. The total efficiencies, integrated over neutrino energy, are shown in the legends and assume a $9.6 \mathrm{M}_{\odot}$ supernova; total efficiencies are higher for the $27 \mathrm{M}_{\odot}$ model.

selected background candidates has been slightly reduced from 0.5 to $0.4 \mathrm{~Hz}$ while the signal efficiency has been increased from $12 \%$ to $44 \%$.

Since the neutrino event classifier is trained on real detector data, no explicit identification of the background components is made. The FD background likely contains significant components from cosmogenic thermal neutron 
captures, cosmogenic ${ }^{12} \mathrm{~B}$ and ${ }^{12} \mathrm{~N}$ beta decays, and singlehit uranium/thorium-chain radioactivity paired with unrelated single-hit electronics noise. The latter is possibly a significant component of the ND background as well, but cosmogenic activity is strongly suppressed compared to the FD.

For 16 of the $40 \mathrm{GW}$ events with ND data, the NuMI beam was in operation. Data at the ND is taken in $5 \mathrm{~ms}$ segments. Any $5 \mathrm{~ms}$ data segment is rejected if it overlaps with a beam pulse or the time up to $3 \mathrm{~ms}$ following a beam pulse. This conservative cut removes all prompt beam activity, muon decays, and neutron captures from thermal neutrons that were produced in the detector and remained in the detector until captured. Some neutron captures can be delayed up to several milliseconds if thermal neutrons spend time in the air surrounding the detector; the $3 \mathrm{~ms}$ cut rejects a large majority of these neutrons.

For each gravitational wave event, we first examine the selected clusters in 1-second bins searching for any significant excess over background, where the background level is determined in situ from the $45 \mathrm{~s}$ readout (or $1000 \mathrm{~s}$ window in the case of FD pulser data). Second, we assume that a supernova burst begins at the gravitational wave time stamp and set limits on its strength for the case of the Garching $9.6 \mathrm{M}_{\odot}$ and $27 \mathrm{M}_{\odot}$ models. Because NOvA's efficiency rises rapidly with neutrino energy between 10 and $30 \mathrm{MeV}$, the higher neutrino energies in the $27 \mathrm{M}_{\odot}$ model result in stronger fluence limits.

Depending on the state of the two NOvA detectors and whether a trigger was received from LVC, several different types of data sets can be available. The best case is when a timely trigger was received and we read $45 \mathrm{~s}$ of continuous data from the ND and FD. In this case, a joint analysis is done using the data from the two detectors. The FD provides more statistical power, but the ND still makes a significant contribution. In some cases, continuous data is available from the ND, but only pulser data from the FD. Again, a joint analysis is performed, but in this case, the ND provides nearly all the statistical power. In some cases, the continuous data from ND or FD is not a complete $45 \mathrm{~s}$, but in all those cases enough was read out to establish the background level and allow the analysis to be run without modification. The background level is not determined with as much precision in these cases, leading to a slight weakening of limits. Finally, in some cases, data from only one detector is available. The status for all GW events is shown in Table I.

\section{RESULTS}

No excess over background is observed for any gravitational wave event at any time within the analyzed window. Background rates were stable at both detectors, being around $5 \mathrm{~Hz}$ at the FD for the continuous-readout selection, $0.3 \mathrm{~Hz}$ for the FD pulser selection and $0.4 \mathrm{~Hz}$ at the ND. Assuming all selected clusters are background, the limits depend on statistical fluctuations in the background
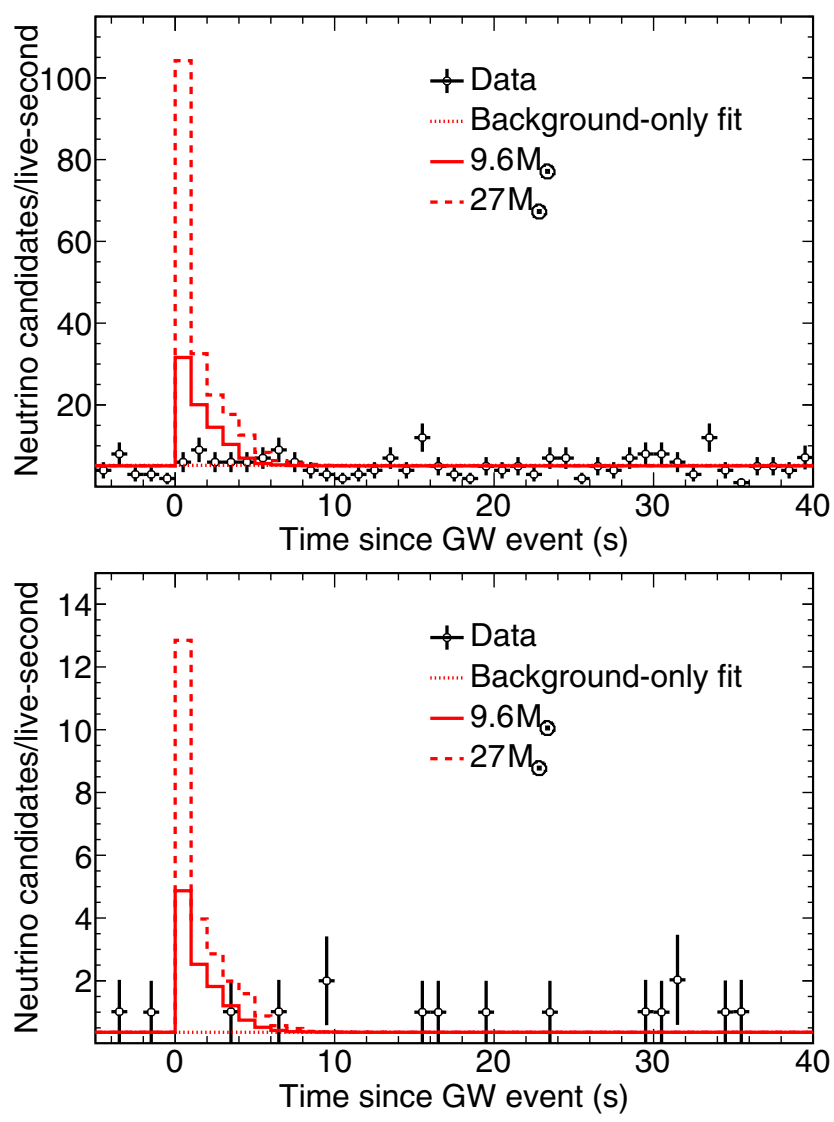

FIG. 2. A typical GW event with both FD (top) and ND (bottom) continuous readout, S200213t. The two supernova models are shown, normalized to $10 \mathrm{kpc}$. The number of neutrino candidates per second is corrected for live time, which is slightly under $100 \%$ in the ND because of beam removal, and in the final bins because readout ends at $39.86 \mathrm{~s}$. The limits set are weaker than the median case because of a slight excess in the $0-5 \mathrm{~s}$ bins in the statistically dominant FD.

in the first few seconds after the gravitational wave time stamp. A typical event is shown in Fig. 2.

For each GW event, $90 \%$ C.L. limits are set on the fluence of the sum of all neutrino flavors, $F$, under the assumption of the two Garching supernova models discussed above, without flavor-changing effects. The limits are set via a fit to the time series of neutrino candidates with two parameters: the background rate and the signal strength, with the signal templates as shown in Fig. 2. A Bayesian approach is used with flat priors in each parameter.

A posterior probability density function, profiled over the background level, is constructed by scanning over signal strength, relative to the prediction at $10 \mathrm{kpc}$, in steps of $10^{-3}$. At each step, the binned log-likelihood,

$$
-\log L=\sum_{\mathrm{i}}\left(m_{i}-d_{i}+d_{i} \log \frac{d_{i}}{m_{i}}\right)
$$

is computed for the background normalization that minimizes $-\log L$, where $m_{i}$ is the number of events predicted 
by the model in bin $i$ and $d_{i}$ is the number of events observed in bin $i$. The probability density is proportional to $L$. The resulting curve is integrated numerically up to $90 \%$ of the total, and this sets the $90 \%$ upper limit on signal strength, $s_{90}$. Because the signal would decrease as $1 / r^{2}$, where $r$ is the distance to the hypothetical supernova, the $90 \%$ lower limit on distance is $r_{90}=10 \mathrm{kpc} / \sqrt{s_{90}}$. Given the number of neutrinos predicted by each model, $N=$ $6.8(11) \times 10^{57}$ for $9.6(27) \mathrm{M}_{\odot}$, fluence limits, $F_{90}$, are related to the distance limits via

$$
F_{90}=\frac{N}{4 \pi r_{90}^{2}} .
$$

No systematic effects are explicitly included in the procedure, but as detailed in Sec. IV, our estimate of the rate of detectable neutrino interactions is conservative; we believe this conservatism is sufficient to cover any systematic effects in signal efficiency. All limits are shown in Table I and a discussion of notable features thereof follows.

For gravitational wave events in which we read out continuous FD data in response to an LVC trigger (the best case), fluence limits range between $F<4 \times 10^{10} \mathrm{~cm}^{-2}$ and $F<15 \times 10^{10} \mathrm{~cm}^{-2}$, assuming the $9.6 \mathrm{M}_{\odot}$ Garching model. In this model, $22 \%$ of the neutrinos are $\bar{\nu}_{\mathrm{e}}$, to which NOvA is primarily sensitive. The median limit is $7 \times 10^{10} \mathrm{~cm}^{-2}$. Similarly, for the $27 \mathrm{M}_{\odot}$ model, in which $23 \%$ of the flux is $\bar{\nu}_{\mathrm{e}}$, we set limits ranging from $F<$ $2.1 \times 10^{10} \mathrm{~cm}^{-2}$ to $F<9 \times 10^{10} \mathrm{~cm}^{-2}$ with a median of $4 \times 10^{10} \mathrm{~cm}^{-2}$. If interpreted as limits on the distance to a hypothetical supernova, we exclude a $9.6 \mathrm{M}_{\odot}$ supernova in the median case, at $90 \%$ C.L., closer than $29 \mathrm{kpc}$. For the event with the strongest exclusion, $\mathrm{S} 200115 \mathrm{j}$, we exclude a $9.6 \mathrm{M}_{\odot}$ supernova closer than $40 \mathrm{kpc}$. For the $27 \mathrm{M}_{\odot}$ model, we exclude a supernova, in the median case, closer than $50 \mathrm{kpc}$, and for $\mathrm{S} 200115 \mathrm{j}, 70 \mathrm{kpc}$.

In the next best case, we have continuous ND data, but either have no FD data or only pulser data from the FD data. In the latter case, the limit is strongly dominated by the ND data. Fluence limits for the $9.6 \mathrm{M}_{\odot}$ model range from $F<19 \times 10^{10} \mathrm{~cm}^{-2}$ to $F<29 \times 10^{10} \mathrm{~cm}^{-2}$, and for the $27 \mathrm{M}_{\odot}$ from $F<13 \times 10^{10} \mathrm{~cm}^{-2}$ to $F<$ $22 \times 10^{10} \mathrm{~cm}^{-2}$. Because of the ND's lower background, the efficiency for selecting lower-energy neutrinos is higher than the FD. The flux model therefore has less effect on fluence limits dominated by ND data. The median distance limit for a $9.6(27) \mathrm{M}_{\odot}$ supernova is $16(24) \mathrm{kpc}$.

Finally, when using only FD pulser data, fluence limits range from $F<190 \times 10^{10} \mathrm{~cm}^{-2}$ to $F<700 \times 10^{10} \mathrm{~cm}^{-2}$ for the $9.6 \mathrm{M}_{\odot}$ model and from $F<110 \times 10^{10} \mathrm{~cm}^{-2}$ to $F<400 \times 10^{10} \mathrm{~cm}^{-2}$ for the $27 \mathrm{M}_{\odot}$ model. Even with only FD pulser data, some exclusion of supernovae in or behind the Galactic core (at $\sim 8 \mathrm{kpc}$ ), whose optical signal may have been obscured, is possible, with distance limits ranging from 2.9-6 kpc for the $9.6 \mathrm{M}_{\odot}$ case and 5-9 kpc for the $27 \mathrm{M}_{\odot}$ case.

The $26 \mathrm{GW}$ events analyzed in our previous report are reanalyzed using the improved analysis. The limits quoted for the seven previously analyzed events that include FD and/or ND continuous readout are now stronger, in the median case, by a factor of 3 , and in no case is the result we now give weaker than our previously published result. However, for events with only FD pulser data, the new analysis techniques only yield a $40 \%$ improvement in fluence limits. There are four GW events that, in the new analysis, have a weaker limit for at least one of the two supernova models: GW170608, GW170729, GW170823 and GW190521_074359. This is an expected consequence of using an analysis that is almost entirely different than our previous analysis, such that there is little correlation between the hits selected previously and now.

\section{CONCLUSIONS}

We have searched for supernovalike neutrinos coincident with 76 gravitational wave events reported by LVC. No excess consistent with such neutrinos was found. Assuming a burst of supernovalike neutrinos beginning at LVC's reconstructed gravitational wave time, we set limits on the fluence of supernovalike neutrinos under two supernova models. In the 32 cases with full FD data, these limits are sufficient to largely exclude the possibility that any of the gravitational waves originated from a stellar core collapse in our Galaxy. This includes the "failed supernovae" in which there is no explosion and/or scenarios that lead to early black hole formation, since similar neutrino luminosities are expected in any of these cases $[48,49]$. Our search complements those performed by other neutrino observatories [10,50-56]. The NOvA detectors will continue to operate for several years, including during the upcoming $\mathrm{O} 4$ run of $\mathrm{LVC}$.

\section{ACKNOWLEDGMENTS}

This document was prepared by the NOvA Collaboration using the resources of the Fermi National Accelerator Laboratory (Fermilab), a U.S. Department of Energy, Office of Science, HEP User Facility. Fermilab is managed by Fermi Research Alliance, LLC (FRA), acting under Contract No. DE-AC02-07CH11359. This work was supported by the U.S. Department of Energy; the U.S. National Science Foundation; the Department of Science and Technology, India; the European Research Council; the MSMT CR, GA UK, Czech Republic; the RAS, RFBR, the Ministry of Education and Science of the Russian Federation (RMES), RSF, and BASIS Foundation, Russia; CNPq and FAPEG, Brazil; STFC, UKRI, and the Royal Society, United Kingdom; and the State and University of Minnesota. We are grateful for the contributions of the staffs of the University of Minnesota at the Ash River Laboratory and of Fermilab. 
[1] B. P. Abbott et al. (LIGO Scientific Collaboration and Virgo Collaboration), GW170817: Observation of Gravitational Waves from a Binary Neutron Star Inspiral, Phys. Rev. Lett. 119, 161101 (2017).

[2] A. Goldstein et al., An ordinary short gamma-ray burst with extraordinary implications: Fermi-GBM detection of GRB 170817A, Astrophys. J. 848, L14 (2017).

[3] B. P. Abbott et al. (LIGO Scientific, Virgo, Fermi-GBM, INTEGRAL Collaborations), Gravitational waves and gamma-rays from a binary neutron star merger: GW170817 and GRB 170817A, Astrophys. J. 848, L13 (2017).

[4] R. Abbott et al. (LIGO Scientific Collaboration, Virgo Collaboration), GWTC-2: Compact Binary Coalescences Observed by LIGO and Virgo During the First Half of the Third Observing Run, Phys. Rev. X 11, 021053 (2021).

[5] B. P. Abbott et al. (LIGO Scientific Collaboration, Virgo Collaboration), A first targeted search for gravitationalwave bursts from core-collapse supernovae in data of firstgeneration laser interferometer detectors, Phys. Rev. D 94, 102001 (2016).

[6] S. M. Adams, C. S. Kochanek, J. F. Beacom, M. R. Vagins, and K.Z. Stanek, Observing the next galactic supernova, Astrophys. J. 778, 164 (2013).

[7] M. A. Acero et al. (NOvA Collaboration), Search for multimessenger signals in NOvA coincident with LIGO/ Virgo detections, Phys. Rev. D 101, 112006 (2020).

[8] D.S. Ayres et al. (NOvA Collaboration), The NOvA technical design report, Report No. FERMILAB-DESIGN-2007-01, 2007.

[9] B. Baret et al., Bounding the time delay between highenergy neutrinos and gravitational-wave transients from gamma-ray bursts, Astropart. Phys. 35, 1 (2011).

[10] S. Adrian-Martinez et al. (Virgo, IceCube, ANTARES, LIGO Scientific Collaborations), High-energy neutrino follow-up search of gravitational wave event GW150914 with ANTARES and IceCube, Phys. Rev. D 93, 122010 (2016).

[11] P. Adamson et al., The NuMI Neutrino Beam, Nucl. Instrum. Methods Phys. Res., Sect. A 806, 279 (2016).

[12] B. P. Abbott et al. (LIGO Scientific Collaboration, Virgo Collaboration), GWTC-1: A Gravitational-Wave Transient Catalog of Compact Binary Mergers Observed by LIGO and Virgo During the First and Second Observing Runs, Phys. Rev. X 9, 031040 (2019).

[13] LIGO Scientific Collaboration and Virgo Collaboration, S190510g, GCN Circular 24442 (2019).

[14] LIGO Scientific Collaboration and Virgo Collaboration, S190718y, GCN 25087 (2019).

[15] LIGO Scientific Collaboration and Virgo Collaboration, S190901ap, GCN 25606 (2019).

[16] LIGO Scientific Collaboration and Virgo Collaboration, S190910d, GCN 25695 (2019).

[17] LIGO Scientific Collaboration and Virgo Collaboration, S190910h, GCN 25707 (2019).

[18] LIGO Scientific Collaboration and Virgo Collaboration, S190923y, GCN 25814 (2019).

[19] LIGO Scientific Collaboration and Virgo Collaboration, S190930t, GCN 25876 (2019).
[20] LIGO Scientific Collaboration and Virgo Collaboration, S191105e, GCN 26182 (2019).

[21] LIGO Scientific Collaboration and Virgo Collaboration, S191109d, GCN 26202 (2019).

[22] LIGO Scientific Collaboration and Virgo Collaboration, S191129u, GCN 26303 (2019).

[23] LIGO Scientific Collaboration and Virgo Collaboration, S191204r, GCN 26334 (2019).

[24] LIGO Scientific Collaboration and Virgo Collaboration, S191205ah, GCN 26350 (2019).

[25] LIGO Scientific Collaboration and Virgo Collaboration, S191213g, GCN 26402 (2019).

[26] LIGO Scientific Collaboration and Virgo Collaboration, S191215w, GCN 26441 (2019).

[27] LIGO Scientific Collaboration and Virgo Collaboration, S191216ap, GCN 26454 (2019).

[28] LIGO Scientific Collaboration and Virgo Collaboration, S191222n, GCN 26543 (2019).

[29] LIGO Scientific Collaboration and Virgo Collaboration, S200105ae, GCN 26640 (2020).

[30] LIGO Scientific Collaboration and Virgo Collaboration, S200112r, GCN 26715 (2020).

[31] LIGO Scientific Collaboration and Virgo Collaboration, S200114f, GCN 26734 (2020).

[32] LIGO Scientific Collaboration and Virgo Collaboration, S200115j, GCN 26759 (2020).

[33] LIGO Scientific Collaboration and Virgo Collaboration, S200128d, GCN 26906 (2020).

[34] LIGO Scientific Collaboration and Virgo Collaboration, S200129m, GCN 26926 (2020).

[35] LIGO Scientific Collaboration and Virgo Collaboration, S200208q, GCN 27014 (2020).

[36] LIGO Scientific Collaboration and Virgo Collaboration, S200213t, GCN 27042 (2020).

[37] LIGO Scientific Collaboration and Virgo Collaboration, S200219ac, GCN 27130 (2020).

[38] LIGO Scientific Collaboration and Virgo Collaboration, S200224ca, GCN 27184 (2020).

[39] LIGO Scientific Collaboration and Virgo Collaboration, S200225q, GCN 27193 (2020).

[40] LIGO Scientific Collaboration and Virgo Collaboration, S200302c, GCN 27278 (2020).

[41] LIGO Scientific Collaboration and Virgo Collaboration, S200311bg, GCN 27358 (2020).

[42] LIGO Scientific Collaboration and Virgo Collaboration, S200316bj, GCN 27388 (2020).

[43] A. Mirizzi, I. Tamborra, H. Janka, N. Saviano, K. Scholberg, R. Bollig, L. Hudepohl, and S. Chakraborty, Supernova neutrinos: Production, oscillations and detection, Riv. Nuovo Cimento 39, 1 (2016).

[44] C. Andreopoulos et al., The GENIE neutrino Monte Carlo generator, Nucl. Instrum. Methods Phys. Res., Sect. A 614, 87 (2010).

[45] S. Agostinelli et al., GEANT4-a simulation toolkit, Nucl. Instrum. Methods Phys. Res., Sect. A 506, 250 (2003).

[46] F. Pedregosa et al., Scikit-learn: Machine learning in PYTHON, J. Mach. Learn. Res. 12, 2825 (2011).

[47] G. Punzi, Sensitivity of searches for new signals and its optimization, eConf C030908, MODT002 (2003). 
[48] A. da Silva Schneider, E. O'Connor, E. Granqvist, A. Betranhandy, and S.M. Couch, Equation of state and progenitor dependence of stellar-mass black hole formation, Astrophys. J. 894, 4 (2020).

[49] S. W. Li, L. F. Roberts, and J. F. Beacom, Exciting prospects for detecting late-time neutrinos from core-collapse supernovae, Phys. Rev. D 103, 023016 (2021).

[50] M. Agostini et al., A search for low-energy neutrinos correlated with gravitational wave events GW150914, GW151226 and GW170104 with the Borexino detector, Astrophys. J. 850, 21 (2017).

[51] A. Gando et al. (KamLAND Collaboration), Search for electron antineutrinos associated with gravitational wave events GW150914 and GW151226 using KamLAND, Astrophys. J. 829, L34 (2016).

[52] S. Abe et al. (KamLAND Collaboration), Search for lowenergy electron antineutrinos in KamLAND associated with gravitational wave events, Astrophys. J. 909, 116 (2021).
[53] K. Abe et al. (Super-Kamiokande), Search for neutrinos in Super-Kamiokande associated with gravitational wave events GW150914 and GW151226, Astrophys. J. 830, L11 (2016).

[54] K. Abe et al. (Super-Kamiokande Collaboration), Search for neutrinos in coincidence with gravitational wave events from the LIGO-Virgo O3a observing run with the SuperKamiokande detector, arXiv:2104.09196.

[55] A. Albert et al. (Virgo, IceCube, ANTARES, LIGO Scientific Collaborations), Search for high-energy neutrinos from gravitational wave event GW151226 and candidate LVT151012 with ANTARES and IceCube, Phys. Rev. D 96, 022005 (2017).

[56] A. Aab et al. (Pierre Auger Collaboration), Ultrahighenergy neutrino follow-up of gravitational wave events GW150914 and GW151226 with the Pierre Auger Observatory, Phys. Rev. D 94, 122007 (2016). 